\title{
Urgences
}

\section{Jouissance de l'autre ou une autre jouissance?}

\section{Pierre-Paul Parent}

Numéro 14, août 1986

Corps et jouissances

URI : https://id.erudit.org/iderudit/025267ar

DOI : https://doi.org/10.7202/025267ar

Aller au sommaire du numéro

Éditeur(s)

Urgences

ISSN

0226-9554 (imprimé)

1927-3924 (numérique)

Découvrir la revue

Citer cet article

Parent, P.-P. (1986). Jouissance de l'autre ou une autre jouissance ? Urgences, (14), 65-71. https://doi.org/10.7202/025267ar d'utilisation que vous pouvez consulter en ligne.

https://apropos.erudit.org/fr/usagers/politique-dutilisation/ 


\section{Pierre-Paul Parent}

Professeur à l'UQAR,

psychologue et analyste, membre du GIFRIC

(Québec) et du groupe de recherche ETHOS (Rimouski)

\section{JOUISSANCE DE L'AUTRE OU UNE AUTRE JOUISSANCE?}


Vouloir parler d'un possible rapport du corps et de la jouissance confine à l'enjeu de l'indicible: c'est donc à partir d'un biais et à l'intérieur d'un cadre précis que je tenterai d'aborder un tel sujet. C'est pourquoi le film américain récent, Neuf semaines et demie (d'Adrian Lyne), servira ici de biais privilégié: il y est donné à "voir" un certain discours sur la jouissance sexuelle. Quant au cadre, il se mettra en place à travers une lecture lacanienne de l'enjeu de l'autre jouissance tel qu'il semble apparaître dans ce film. Je présenterai donc brièvement ce film au lecteur ou à la lectrice; puis, je tenterai d'en faire une lecture de type psychanalytique.

Une jeune femme divorcée, responsable d'une galerie de peinture à New York, rencontre par hasard un jeune homme dont on saura progressivement qu'il a réussi financièrement comme gestionnaire d'opérations boursières'. Les regards se croisent, ils se parlent et l'homme amène la femme chez lui avec l'intention claire de faire l'amour. Ce premier rendez-vous se déroulera sur un mode pseudopervers de type sado masochiste, où la peur sera l'élément prégnant (paroles à l'appui, décor campé d'ue studio près de la mer, isolé, sans téléphone, etc.). $\mathrm{Si}$, prise de panique, elle s'en ira, prétextant du travail, elle n'en attendra pas moins des nouvelles de ce nouvel ami, nouvelles qui lui viendront avec un envoi de fleurs. Se met donc en place un échange où la peur et la sensualité font un ménage plus ou moins harmonieux. Dès le début de leurs relations, elle demande de la part de son amant une parole qui viendrait inscrire leur relation dans une histoire: cette parole ne viendra pas. Les deux se retrouveront à maintes reprises; elle acceptera de "réaliser" le fantasme de l'homme, fantasme qu'il s'efforce d'isoler, de couper de son vécu. Ce fantasme s'exprime à répétition à travers le scénario suivant: pendant qu'elle aura les yeux bandés, ou fermés, alors qu'il la regarde, ils élaboreront progressivement, ou plutôt il élaborera, en utilisant sa maîtresse comme un objet, un jeu de l'amour. Son amante est en quelque sorte campée par lui comme un objet de jouissance, sans regard, alors qu'elle attendrait une parole de sa part. Il s'agit là d'un échange à la fois sensuel et agressif, d'une agressivité cependant contenue, simulée ou évoquée. 
L'expression des fantasmes de l'homme exigera de plus en plus d'être alimentée par des comportements chez son amante qui la renverront à une image négative d'elle-même. Elle demandera d'ailleurs à son amant comment il pouvait savoir qu'elle accepterait de faire ainsi tout ce qu'il lui demandait. Leurs relations, ou ce qu'on pourrait identifier comme tel, connaîtront une telle progression à travers les changements de lieu, tout au long de ces neuf semaines et demie. À travers ce scénario, on peut remarquer que, si l'hornme parvient à bien isoler travail et érotisme, il n'est est pas de même pour elle: sa vie est en quelque sorte complètement altérée, transformée. Se pose la question suivante pour elle: jusqu'où ira-t-elle dans cette abjection à laquelle il la renvoie. Lui apparaît en quelque sorte une vacuité de sa vie de travail, un vide qu'elle n'arrive pas à combler. Un événement anodin en lui-même, mais qui devient très signifiant pour elle, entrera en ligne de compte dans ce parcours. Elle doit entrer en contact avec un peintre qui exposera bientôt à la galerie. Cette rencontre a lieu à la demeure du peintre, ailleurs, un peu dans un espace hors-temps. Que vient-il lui signifier d'un ailleurs? Qu'ouvre-t-il comme béance dans ce parcours, au moment où elle se demande justement jusqu'où elle est prête à aller dans la mise en acte des fantasmes de son amant: cette question est introduite dans cette rencontre.

À la suite de celle-ci, un événement viendra précipiter la fin d'un tel échange sexuel, pour ainsi dire. Lors de la rencontre des amants qui suivra celle du peintre, son amant, qui ne peut plus compter', exige d'elle qu'elle marche à quatre pattes alors qu'il lui lancera des billets au visage. En quête d'amour, dans l'attente de mots qui seraient venus de son amant, elle s'était soumise à ses demandes. De se voir réduite à une position de femme-putain, elle réalise à quel degré d'abjection elle en est réduite. Elle refuse d'obéir à une telle demande et part. À la rencontre suivante, il exige d'elle cette fois de se bander les yeux et de se soumettre aux caresses d'une autre femme, dans un hôtel de passe newyorkais, alors qu'il les regardera faire. Elle ne supportera pas cette réduction à un objet qu'on paie ou qu'on utilise et qu'on jette plus ou moins après usage. Ce moment apparaît comme le point tournant du film. Cet espoir d'une parole qui la rassurerait, qui l'articulerait à une histoire a échoué. "Regarde ce que tu as fait de moi", semblera-t-elle dire à son amant, en se précipitant immédiatement dans un sex-shop des environs et en embrassant le premier 
homme venu, à la vue d'un couple faisant l'amour devant des spectateurs mâles. Son amant arrivant en courant à sa suite, elle mimera en quelque sorte les gestes du couple.

Elle rentre alors chez elle, complètement bouleversée par ces événements. C'est le moment du vernissage de l'exposition de peinture. Le peintre est là, mais il apparaît tout à fait à l'extérieur de cette foule bruyante qui se précipite vers les petits fours. Lui apparaît alors l'évidence d'une confrontation inéluctable à ce vide intérieur qu'elle avait cherché à combler avec son amant. Au terme d'une nuit sans sommeil, elle le quittera en lui disant qu'il est trop tard, alors qu'il tentera pour la première fois de parler de lui, de l'xattacher» à son histoire. Ces paroles viennent trop tard, elle s'en ira seule. Le film se terminera ainsi.

Au terme de la présentation des principaux éléments de ce film, pertinents pour mon projet, une lecture psychanalytique devrait permettre de cerner ce qu'il en est des enjeux de la jouissance dans lesquels sont "pris" en quelque sorte cette femme et cet homme.

Que cherchait cette femme, qu'attendait-elle de cette rencontre? Une première lecture nous permet de voir qu'elle attendait des mots, une parole qui viendrait combler un vide, un trou. Vide, trou auquel le silence de l'homme la renvoyait. Dans la première scène, ne tente-telle pas d'amorcer un échange, de quêter en quelque sorte une assurance nouvelle qui apporterait la parole face à sa peur. Elle est pourtant amenée à ce lieu de vacuité de certaines paroles, celles qui seraient articulées à une histoire, à du temps, bien qu'elle les demande. Elle joue le jeu de cette absence de parole; elle y entre et s'y perd. Car n'était-elle pas, au fond d'elle-même, confrontée à ce vide bien avant qu'elle ne rencontre cet homme, bien avant qu'elle le mette en quelque sorte au défi de combler un tel vide. Et ce sera justement au moment où il commence à parler qu'elle partira. Ne reste-t-elle pas avec lui alors même qu'il en fait un objet de jouissance, alors même qu'elle attend cette position de sujet. Mais, partir au moment même où il la placerait dans cette position de sujet devient peut-être le signe évident qu'elle est confrontée à des signifiants d'un autre ordre et qu'elle ne peut plus se prendre au jeu des "fictions provisoires" qu'il pourrait lui proposer et qui lui rendraient ce vide 
supportable. Elle s'en ira, encore plus convaincue que ce ne peut être qu'ailleurs qu'elle trouvera des signifiants qui la feront sujet. Des signifiants qui, venant de l'inconscient, d'un espace anhistorique, permettraient d'articuler un désir propre.

Quant à l'homme, n'est-il pas un névrosé qui se prend au jeu du pervers? Qui pense tirer une jouissance du corps de l'autre, en isolant bien tous ses repères identificatoires (travail, affaires) du monde du sexuel, réduisant en quelque sorte le sexuel à l'érotisme? Vouloir se maintenir hors du champ de la parole, réduire l'autre au silence, faire du corps de l'autre un objet de jouissance, ce sont autant de moyens qu'il utilisera pour isoler les uns des autres certains des éléments de sa vie. Serait-il un pervers: il me semble qu'il ne se serait pas arrêté alors qu'elle disait que c'était fini et aurait recherché la jouissance dans un acte confinant à la mort. Dénier l'autre jusque dans la mort. Plutôt un névrosé jouant le jeu du pervers: il se prend au jeu de l'autre et, justement, en bon névrosé, passe à la parole au moment où sa maîtresse - nous pourrions dire aussi "la maîtrise" - lui échappe. Au moment où elle s'en va. Il est alors confronté à la perte, perte qu'il ne supporte pas et qu'il pense conjurer magiquement en se disant qu'elle reviendra avant qu'il n'ait fini de compter jusqu'à... Compter pour garder, maintenir.

Allons plus loin et demandons-nous ce que le fait de tenter de tracer à grands traits ces deux "personnalités" en essayant de définir leurs structures, pourrait avoir d'opératoire. En effet, et c'est là un des effets du film, ces personnalités sont présentées ici de façon un peu caricaturale: l'expression des signes, des manifestations névrotiques rend ici la lecture plus facile. Ceci surtout pour l'homme.

Selon l'homme, la femme semble trouver là l'enjeu de la confrontation au vide, à l'insensé, et la tentative de combler ce vide. Cependant, une telle tentative est, on le voit, vouée à l'échec. En cela, elle fait la démonstration d'un enjeu de la féminité. Cette fermme ne peut pas se rassurer dans des conduites sociales appropriées, dans des identifications sociales. Elle voit que de telles conduites sont à juste raison comme de la poudre jetée aux yeux de l'autre, de l'illusion lancée à la face de l'incertitude, des leurres dont elle n'est pas dupe chez les 
autres et encore moins chez elle. Ne pouvant pas s'appuyer sur de telles identifications, elle est sans défense contre la jouissance de l'autre car c'est tout entière dans cette question du rapport à l'autre qu'elle situe ses enjeux de vie. Et pourtant, elle trouvera la vacuité, le vide même de cette parole qu'elle cherchait, parole dont elle attendait au départ qu'elle l'inscrive dans une dimension historique: en effet, au moment où elle entendra une telle parole, elle partira ailleurs. La femme pourra-t-elle accéder à une jouissance non mortifère, à une jouissance d'un autre ordre, marquée cette fois par l'assomption du vide: tel pourrait se définir l'enjeu de la féminité mis de l'avant dans ce film. Une telle attente pourrait trouver sa réponse dans des signifiants la renvoyant à cette impossibilité structurale d'être l'objet de satisfaction totale de l'autre.

L'enjeu de la masculinité, mis en scène par l'homme de ce film, est différent. Celui-ci pense justement pouvoir donner le change, bien isoler jouissance et vie sociale, maintenir solides des identifications sociales tout en les isolant de son rapport à l'autre et à la jouissance de l'autre. Utiliser le corps comme un objet, tant son propre corps que celui de l'autre, en garantissant bien, par ailleurs, son rapport au social. On voit que, si pour la femme l'illusion de la réussite sociale ne peut pas tenir, elle pourra ici fonctionner éventuellement toute une vie sans être remise en question. Ça n'est peut-être que lorsque la personne est confrontée malgré elle au vide et qu'elle est prise au jeu (comme on le voit à la fin de ce film), qu'elle peut alors saisir la faiblesse des garanties qu'elle s'était données.

Ces deux personnages, nous le voyons, sont donc les illustrations de deux rapports différents au corps et à la jouissance. D'une part, un corps livré à la jouissance de l'autre, confronté au vide, dans l'attente d'une parole qui réduirait ce vide intérieur. D'autre part, un corps et une jouissance isolées, qu'on veut surtout maintenir dans un champ restreint du fantasme qu'on agit à répétition.

Je terminerais en ajoutant ceci: à partir de ce film, nous pouvons voir que cette femme est renvoyée à des signifiants qui viennent d'ailleurs, qui n'ont rien à voir avec ce qu'elle attend de cet homme. En effet, c'est bien plutôt à travers l'opacité et le hors-temps représentés par le personnage du peintre qu'elle en prendra conscience. Mais l'homme n'est-il pas aussi confronté, à sa façon, à un tel enjeu? Ce n'est que 
lorsqu'elle est partie qu'il s'adresse à elle, comme quoi c'est bien d'ailleurs, du lieu de l'autre qu'ils peuvent venir baliser une jouissance autre, celle justement qui renvoie à une position de sujet.

1. Cherchant les noms des personnages, je n'ai malheureusement pas pu les retrouver. On peut se demander si justement ici on n'en était pas dans un en-deçă du langage.

2. Est-ce lả pour lui un signe, manifeste d'un déplacement d'impuissance? 